\title{
Programación nutricional en el pejelagarto (Atractosteus tropicus): efecto del almidón de maíz sobre la bioquímica sanguínea
}

\author{
Rocío Guerrero Zárate \\ rocio.guerrero@ujat.mx
}

\section{Ronald Jesús Contreras romeo4500@gmail.com}

\section{Carlos Alfonso Álvarez González alvarez_alfonso@hotmail.com}

Laboratorio de Acuicultura Tropical, División Académica de Ciencias Biológicas, Universidad Juárez Autónoma de Tabasco (UJAT), Carretera Villahermosa-Cárdenas s/n, Km 0, C.P. 86039 Villahermosa,

Tabasco, México.

\section{RESUMEN}

El pejelagarto es una especie carnívora, en la que se ha demostrado una mejora en el crecimiento durante el larvicultivo al incluir almidón de maíz en su dieta, así como una eficiencia en la ruta de la gluconeogénesis al utilizar hasta $23 \%$ de carbohidratos en sus dietas. El objetivo de esta investigación fue determinar si el uso de almidón de maíz en el larvicultivo puede producir un afecto de programación metabólica, determinado mediante la bioquímica sanguínea durante la etapa juvenil. Los peces fueron divididos en 3 grupos, los cuales se alimentaron durante 73 días con las dietas experimentales, que tenían diferente porcentaje de inclusión de almidón de maíz (13.8, 22.5 y 28.9 \% de carbohidratos). Posteriormente, los peces de los 3 grupos fueron sometidos a una prueba de tolerancia a la glucosa y se midió la concentración de glucosa, triglicéridos y colesterol a las $0,1.5,3$ y 5 h después de aplicar una descarga de dextrosa intraperitonealmente. Los resultados demuestran que la concentración de carbohidratos y lípidos suministrada mediante la dieta durante la etapa temprana de crianza puede modificar de forma permanente las concentraciones de glucosa y triglicéridos en plasma de los pejelagartos juveniles.

Palabras clave: pejelagarto; glucosa; almidón maíz; programación metabólica; gluconeogénesis 


\title{
Nutritional programming in tropical gar: effect of cornstarch on blood parameters
}

\begin{abstract}
The tropical gar is a carnivorous species that has shown improvement in growth during larviculture by including cornstarch in its diet, as well as an efficiency in the gluconeogenesis pathway by using up to $23 \%$ carbohydrates in their diets. The objective of this research was to determine if the use of cornstarch in larviculture can produce a metabolic programming effect determined by blood parameters during the juvenile stage. The fish were fed 3 diets with different inclusion of corn starch $(13.8,22.5$ y 28.9 $\%$ carbohydrates) for 73 days. Subsequently, the fish of the 3 groups were subjected to a glucose tolerance test and the concentration of glucose, triglycerides and cholesterol was measured at $0,1.5,3$ and $5 \mathrm{~h}$ after applying a dextrose discharge intraperitoneally. The results demonstrate that the concentration of carbohydrates and lipids supplied through the diet during the early stage of rearing can permanently modify the concentrations of glucose and triglycerides in the plasma of tropical gar juvenile.
\end{abstract}

Keywords: tropical gar; glucose; corn starch; metabolic programming; gluconeogenesis

Artículo recibido: 05 octubre. 2021 Aceptado para publicación: 02 noviembre 2021 Correspondencia: rocio7224@hotmail.com Conflictos de Interés: Ninguna que declarar 


\section{INTRODUCCIÓN}

El rápido crecimiento de la acuacultura a nivel internacional, así como la falta de estabilidad de producción y el incremento en los costos de la harina de pescado han orientado las investigaciones en nutrición acuícola hacia la búsqueda de ingredientes alternativos, varios de estos ingredientes son de origen vegetal por lo que contienen considerables cantidades de carbohidratos (Panserat, Marandel, \& Seiliez, 2019). Así mismo, el uso de carbohidratos digeribles (almidón) aporta ventajas como ligación, flotabilidad y estabilidad de las dietas y disminución de descargas de nitrógeno a los efluentes, además de ser de tres a cinco veces más baratos que las proteínas y lípidos (Kamalam \& Panserat, 2016; Wilson, 1994). En los peces los carbohidratos son importantes debido a que sus tejidos cerebrales, renales, oculares y branquiales utilizan la glucosa como fuente preferente de energía (Polakof, Mommsen, \& Soengas, 2011). A pesar de ello, no tienen un requerimiento específico de carbohidratos debido a que tienen la capacidad de sintetizar glucosa a partir de precursores como lactato, piruvato y aminoácidos (Kamalam, Medale, \& Panserat, 2017). Esta demostrado que la eficiencia en el aprovechamiento de carbohidratos es variable entre especies (Polakof, Panserat, Soengas, \& Moon, 2012), de tal forma que las especies carnívoras muestran una menor tolerancia a la glucosa que las especies omnívoras y herbívoras (Enes, Panserat, Kaushik, \& Oliva-Teles, 2008; Hemre, Mommsen, \& Krogodahl, 2002).

Por otro lado, estudios realizados en mamíferos y humanos demuestran que estímulos aplicados durante un periodo o ventana crítica de tiempo en las etapas tempranas de vida de un organismo pueden tener repercusión a largo plazo en sus funciones fisiológicas en etapas adultas, a esto se le conoce como programación nutricional (Panserat et al., 2019) o programación metabólica (Hou \& Fuiman, 2019). En los peces, el concepto de programación nutricional ha sido recientemente estudiado, y se ha demostrado éxito en especies como el pez cebra (Danio rerio) y el esturión siberiano (Acipenser baerii) (Fang et al., 2014; Gong et al., 2015).

El pejelagarto Atractosteus tropicus es un pez dulceacuícola descrito como carnívoro con capacidad para aprovechar ingredientes alternativos (Frías-Quintana, ÁlvarezGonzález, \& Márquez-Couturier, 2010; Guerrero-Zárate et al., 2014), investigaciones recientes han mostrado que es capaz de mejorar su crecimiento durante la etapa larval al agregar almidón de maíz en sus dietas (Frías-Quintana, Domínguez-Lorenzo, Álvarez- 
González, Tovar-Ramírez, \& Martínez-García, 2016) y además es capaz de regular eficientemente las rutas del metabolismo intermediario en respuesta a la ingestión de dietas hasta con un 23\% de carbohidratos (Guerrero-Zárate et al., 2019). Sin embargo, se desconoce si la inclusión de almidón durante la etapa de larvicultivo tiene repercusiones a largo plazo en el metabolismo de carbohidratos. Por ello, el objetivo de la presente investigación fue determinar si incluir almidón de maíz en su dieta durante la etapa de larvicultivo puede producir un efecto de programación metabólica en la bioquímica sanguínea en la etapa juvenil del pejelagarto $A$. tropicus.

\section{MATERIALES Y MÉTODOS}

\section{Dietas y análisis proximales}

Se formularon tres dietas con diferente proporción de almidón de maíz pregelatinizado utilizando el programa Mixit-Win V.5 (Agricultural Software Consultants, Inc, San Diego, CA). La dieta control (D1) se basó en la formulación con harina de pescado propuesta para pejelagartos en etapa larval (Frías-Quintana et al., 2010). Las otras dos dietas se diseñaron con incrementos graduales del 50\% de almidón de maíz pregelatinizado mientras se disminuía la cantidad de lípidos añadidos a la dieta (Tabla $1)$.

Tabla 1. Composición química y análisis proximal de las dietas experimentales

\begin{tabular}{|c|c|c|c|}
\hline Ingredientes $\left(\mathrm{g} \mathrm{Kg}^{-1}\right)$ & $\begin{array}{c}\text { D1 (100\%L/ } 0 \% \\
\text { CHO) }\end{array}$ & $\begin{array}{l}\text { D2 (50\%L/ } \\
50 \% \text { CHO) }\end{array}$ & $\begin{array}{c}\text { D3(0\% L/ } 100 \\
\text { CHO) }\end{array}$ \\
\hline Harina de sardina ${ }^{1}$ & 546 & 546 & 546 \\
\hline Aceite de sardina ${ }^{1}$ & 72 & 35 & 0 \\
\hline Hidrolizado de pescado ${ }^{1}$ & 100 & 100 & 100 \\
\hline Lecitina de soya $^{2}$ & 36 & 18 & 0 \\
\hline $\begin{array}{l}\text { Almidón de maíz } \\
\text { pregelatinizado }^{3}\end{array}$ & 0 & 125 & 250 \\
\hline Grenetina $^{6}$ & 20 & 20 & 20 \\
\hline $\begin{array}{l}\text { Premezcla de vitaminas y } \\
\text { minerales }^{7}\end{array}$ & 15 & 15 & 15 \\
\hline Vitamina $\mathrm{C}^{4}$ & 0.8 & 0.8 & 0.8 \\
\hline Harina de sorgo $8-10 \% 5$ & 210 & 140 & 68 \\
\hline Materia seca & 975 & 983 & 976 \\
\hline Proteína cruda & 425 & 418 & 415 \\
\hline Lípidos & 183 & 107 & 62.4 \\
\hline Carbohidratos & 138 & 225 & 289 \\
\hline Cenizas & 98 & 114 & 118 \\
\hline $\begin{array}{l}\text { Proporción carbohidratos: } \\
\text { lípidos }\end{array}$ & 0.75 & 2.10 & 4.63 \\
\hline
\end{tabular}


${ }^{1}$ Proteinas Marinas y Agropecuarias, S.A. de C.V., Guadalajara, Jalisco, México.

${ }^{2}$ Pronat Ultra, Mérida, Yucatán, México. ${ }^{3}$ MSA Industrializadora de Maíz, S.A. de C.V., Guadalajara, Jalisco, México ${ }^{4}$ DMS (Heerlen, Netherlands, active agent 35\%). ${ }^{5}$ GALMEX Comercializadora de Insumos Agrícolas, Villahermosa, Tabasco, México. ${ }^{6}$ D'gari Productos alimenticios y dietéticos Relámpago, Tlalpan, Edomex, México. ${ }^{7}$ Consorcio Súper Sociedad Anónima de Capital Variable. (para trucha), Guadalajara, Jalisco, México.

Para la elaboración de las dietas experimentales, los macronutrientes fueron molidos, cribados y mezclados. A esta mezcla se añadieron los micronutrientes (vitaminas y minerales). Posteriormente se agregaron los ingredientes líquidos (aceite de pescado y lecitina de soya) y finalmente se añadió agua. Las mezclas fueron peletizadas y secadas a $50^{\circ} \mathrm{C}$ por $10 \mathrm{~h}$. Después fueron trituradas y cribadas para cubrir el rango de tamaño de partícula requerido durante la crianza larvaria $(250-1000 \mu \mathrm{m})$. Las dietas fueron almacenadas en refrigeración a $-20^{\circ} \mathrm{C}$.

La composición proximal de las dietas fue determinada de acuerdo a la Association of Official Analytical Chemist procedures (William Horwitz, 2000). La humedad fue calculada por gravimetría después de secar las muestras a $105^{\circ} \mathrm{C}$ por $24 \mathrm{~h}$. El contenido de proteína cruda fue determinado por el método Kjendahl usando un sistema de destilación automática VAPODEST 10s, (Gerhardt, Königswinter, Alemania) y un sistema de titulación automática TitroLine® 5000 (SI Analytics, College Station, TX). Los lípidos totales fueron determinados por la extracción con metanol-cloroformo (Folch, Lees, \& Sloan Stanley, 1957). El almidón fue determinado por el método con amilasa y glucosa oxidasa (Thivend, Mercier, \& Guilbot, 1972). El contenido de cenizas totales fue determinado por gravimetría después de la combustión de las muestras a 550 ${ }^{\circ} \mathrm{C}$ por 8 h (Tabla 7).

\section{Sistema de cultivo}

El sistema experimental utilizado era parte de un sistema de recirculación con temperatura controlada de $28.9 \pm 1.2{ }^{\circ} \mathrm{C}$. El sistema contaba también con filtración mecánica y biológica y oxígeno suplementario. Los peces fueron mantenidos bajo un fotoperiodo natural (12:12). Los parámetros de calidad de agua fueron monitoreados durante el periodo experimental: el $\mathrm{pH}$ fue de $8.0 \pm 0.4$ ( $\mathrm{pH}$ pen meter ST10, Ohaus, 
Parsippany, NJ), el oxígeno disuelto de $5.1 \pm 0.5 \mathrm{mg} \mathrm{L}^{-1}$ (DO instrument, YSI 55-12FT, Yellow Springs, OH) el nitrito y amonio de $0.19 \pm 0.13 \mathrm{mg} \mathrm{L}^{-1}$ (Ammonia Test Kit, Mars Fishcare, Chalfont, PA).

\section{Crianza de organismos y procedimientos experimentales}

Las larvas de pejelagarto fueron obtenidas a partir de un desove inducido en una hembra y 3 machos de A. tropicus, en el Laboratorio de Acuicultura Tropical ubicado en la División Académica de Ciencias Biológicas de la Universidad Juárez Autónoma de Tabasco. Una vez que las larvas iniciaron el nado (4 días después de la eclosión, DDE) se transfirieron a tinas con volumen de $70 \mathrm{~L}$ conectadas a un sistema de recirculación con temperatura controlada. Las larvas fueron adaptadas a las dietas experimentales por una coalimentación con nauplios de artemia (4-13 DDE) y posteriormente con biomasa congelada de artemia adulta (14-17 DDE) (Márquez-Couturier, Vázquez-Navarrete, Contreras-Sánchez, \& Alvarez-González, 2015), después de este periodo se les suministraron únicamente las dietas experimentales.

Al inicio del experimento un total de 360 larvas de pejelagarto, con peso inicial de 0.03 $\pm 0.002 \mathrm{~g}$ y longitud de $1.76 \pm 0.05 \mathrm{~cm}$, fueron divididas en 3 grupos, a razón de 120 peces por tina. A cada tina se le asignó uno de los tres tratamientos (D1, D2 o D3). Los peces fueron alimentados con sus respectivas dietas 4 veces por día (8:00, 11:00, 14:00 y 17:00) a saciedad aparente durante 73 días con las dietas experimentales (77DDE). Posteriormente, se alimentaron los peces de los 3 grupos con alimento comercial para trucha (Silver Cup, El pedregal, proteína 52\%, lípidos 16\%) durante 24 días más (97 DDE), (Figura 1).

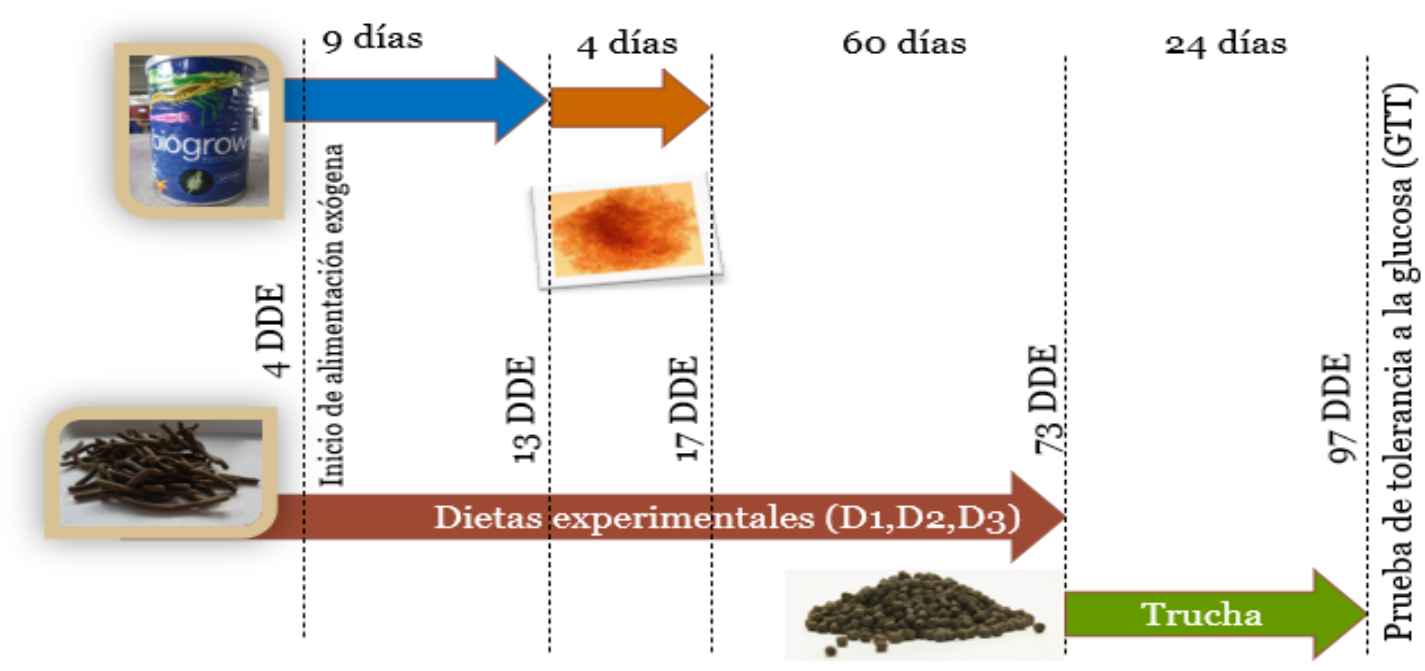

Figura 1. Esquema de alimentación aplicado a los organismos experimentales previo a ser sometidos a la prueba de tolerancia a la glucosa (GTT) 


\section{Prueba de tolerancia a la glucosa (GTT)}

Transcurrido este tiempo, los peces de los 3 grupos que recibieron las dietas experimentales fueron pesados y se verificó su estado de salud. De cada grupo se seleccionaron 36 peces, con peso homogéneo $(22 \pm 4.6 \mathrm{~g})$ y con características de salud adecuadas (sin heridas, aletas rotas, indicios de agresión) y fueron asignados al azar en 12 unidades experimentales, a razón de 3 peces por tina. Cada uno de los tratamientos $(0,1.5,3$ y $5 \mathrm{~h}$ después de la inyección de glucosa) fueron asignados al azar a las unidades experimentales, por triplicado. Finalmente se tuvo un diseño bifactorial $(3 \mathrm{x}$ 4), con los factores dieta (D1, D2 y D3) y tiempo después de la aplicación de glucosa $(0,1.5,3$ y $5 \mathrm{~h})$, cada uno evaluado por triplicado. Los peces fueron sometidos a $24 \mathrm{~h}$ de ayuno. Posteriormente se realizó la GTT, para ello los peces fueron anestesiados con aceite de clavo $(0.1 \mathrm{ml} / \mathrm{L})$, cada pez fue inyectado con jeringas de insulina intraperitonealmente con dextrosa ( $1.2 \mathrm{~g} / \mathrm{kg}$ de peso) y devuelto al contenedor que le correspondía. De acuerdo con cada tiempo establecido por el tratamiento asignado. Transcurrido este tiempo, se tomó una muestra individual de $200 \mu 1$ de sangre mediante punción de la vena caudal con jeringas de insulina. La sangre fue preservada en microtubos con EDTA e inmediatamente centrifugada a $10000 \times g$ por $1 \mathrm{~min}$, las muestras individuales de plasma fueron analizadas (el mismo día de la extracción) para determinar la concentración de glucosa, triglicéridos y colesterol en plasma.

\section{Análisis de química sanguínea}

Los metabolitos en plasma fueron analizados utilizando kits comerciales de Pointe scientific (Michigan, USA) adaptados a microplacas (xMark ${ }^{\mathrm{TM}}$ Microplate Absorbance Spectrophotometer, Bio-Rad, California, USA). La glucosa en plasma fue determinada por el método de glucosa oxidase (G7521), los triglicéridos por reacción enzimática usando glicerol fosfato oxidasa (T7532), y el colesterol fue determinado usando las enzimas colesterol esterasa y colesterol oxidasa (C7510).

\section{Análisis estadísticos}

Los datos fueron analizados estadísticamente mediante un ANOVA de 2 vías, previa verificación de los supuestos de normalidad y homocedasticidad. Donde se encontraron diferencias significativas se realizó la prueba a posteriori de Tukey para determinar las medias estadísticamente diferentes. Los análisis fueron realizados con el software Statistica TM v.8.0 (Statsoft) utilizando un valor de significancia de 0.05. 


\section{RESULTADOS Y DISCUSIÓN}

Los resultados muestran que la concentración de glucosa en plasma se ve significativamente afectada tanto por la dieta suministrada al inicio de la alimentación $(\mathrm{P}=0.005)$ como por el tiempo transcurrido después de aplicar una carga de dextrosa $(\mathrm{P}=$ 0.004). La concentración de glucosa en plasma tiende a ser menor en los peces alimentados con la dieta $2(22 \%$ de carbohidratos), mientras las mayores concentraciones de glucosa se observan en los peces alimentados con la D3 (29\% de carbohidratos) (Figura 2). En cuanto al tiempo después del tratamiento, la menor concentración de glucosa se observa en el tiempo 0 y la mayor concentración se observó $1.5 \mathrm{~h}$ después del tratamiento. Los tiempos 3 y $5 \mathrm{~h}$ posteriores al tratamiento son estadísticamente semejantes a los tiempos 0 y $1.5 \mathrm{~h}$.

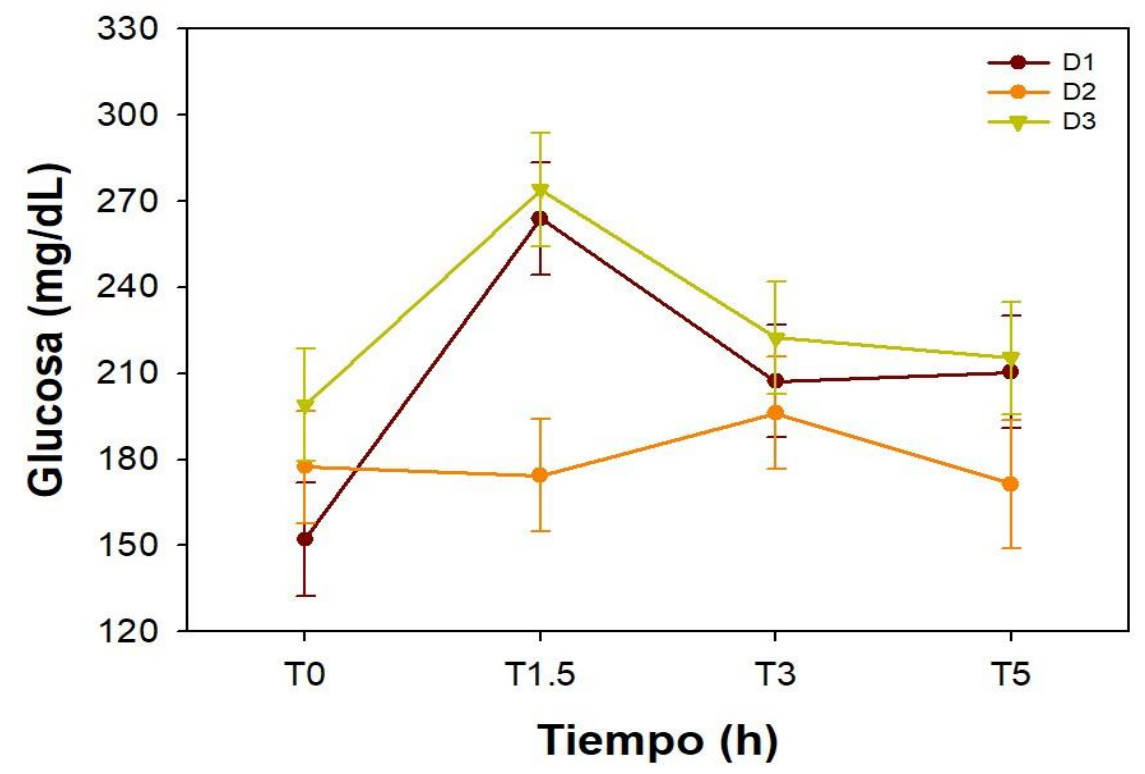

Figura 2. Niveles de glucosa en plasma a diferentes tiempos después de la administración de $1.2 \mathrm{~g}$ de dextrosa/kg de peso. Media \pm EE, $n=9$

Estos resultados sugieren una posible programación temprana para una mejora para la utilización de carbohidratos en la etapa adulta evidenciada por una disminución de la concentración de los niveles de glucosa en plasma en los peces alimentados con la D2 (22\% CHO) en comparación a los peces alimentados con la D1 y D3 y sometidos posteriormente a la prueba de tolerancia a la glucosa.

Resultados similares fueron descritos en el esturión siberiano. Gong et al. (2015) señalan que en A.baerii la glicemia posprandial en adultos puede ser mejorada por un 
estímulo con carbohidratos aplicado durante la etapa larval. Los peces que recibieron dietas ricas en carbohidratos en la etapa larval mostraron niveles más altos de glucosa en plasma a las 3 y 6 horas después de la última alimentación cuando eran adultos. Pero, a las 12 y 24 h después de la última alimentación, el grupo de peces que recibió el estímulo nutricional tuvo niveles de glucosa en plasma menores que los peces alimentados con dietas libres de carbohidratos.

Así mismo, Liang et al. (2017), quienes realizaron un experimento en el que alimentaron un grupo de esturiones (A. baerii) durante su primera etapa de desarrollo con una dieta rica en carbohidratos (57\% de glucosa) y tuvieron otro grupo como control al que alimentaron con una dieta baja en carbohidratos (3.6 \% $\mathrm{CHO})$. Posteriormente, dividieron estos grupos y los sometieron a dietas con un alto (35\%) y bajo contenido (3.6\%) de carbohidratos, seguido de un periodo de inanición y realimentación. Sus resultados muestran que tanto un estímulo en etapas tempranas con glucosa, como el nivel de carbohidratos presente en la dieta posterior al estímulo nutricional, afecto la respuesta de la gluconeogénesis en los periodos de inanición y realimentación. Los peces que recibieron el estímulo temprano de una dieta alta en carbohidratos mostraron menor capacidad para regular la gluconeogénesis (baja actividad de las enzimas PEPCK y FBPasa durante la inanición). También encontraron que los esturiones siberianos pueden controlar el nivel de glucosa en plasma durante los periodos de inanición y realimentación con dietas altas o bajas en carbohidratos.

Un factor común en las especies de peces intolerantes a la glucosa es la falta de regulación de la gluconeogénesis cuando se les suministran carbohidratos, se ha demostrado en diversas especies que la administración de carbohidratos (de forma oral o intraperitoneal) no disminuye la actividad de enzimas o la expresión de genes clave de la gluconeogénesis, entre ellas la fosfoenolpiruvato carboxiquinasa (PEPCK) y la glucosa 6 fosfatasa (G6Pasa) (Enes, Panserat, Kaushik, \& Oliva-Teles, 2006; Enes et al., 2008; Panserat, Médale, Blin, et al., 2000; Panserat, Médale, Brèque, Plagnes-Juan, \& Kaushik, 2000; Polakof et al., 2011; Polakof, Skiba-Cassy, \& Panserat, 2009).

Un estudio realizado en el pez cebra (D. rerio) reveló que dietas ricas en carbohidratos administradas en la etapa larval pueden disminuir la concentración de glucosa en plasma en los organismos adultos, así como la expresión del gen que codifica para la enzima gluconeogénica PEPCK, de tal forma que los autores sugieren que la ruta de la 
gluconeogénesis puede ser controlada mediante la programación nutricional con carbohidratos. Además, concluyen que la ventana critica se sitúa entre la apertura de la boca y la alimentación exógena exclusiva (sin aporte de vitelo), lo que permite modificar permanentemente el metabolismo de los peces cebra adultos por una programación nutricional temprana (Fang et al., 2014).

En la presente investigación no se midieron los niveles de actividad de enzimas clave en la regulación del metabolismo intermediario (rutas de la glucólisis, gluconeogénesis), pero una investigación previa realizada en pejelagartos muestra que en los peces alimentados con dietas con hasta $22 \%$ de carbohidratos disminuye la actividad de las enzimas PEPCK y fructosa-1,6-bifosfatasa, que son clave en la ruta de la gluconeogénesis. Sin embargo, una dieta con $28 \%$ de carbohidratos tiende a incrementar la actividad de estas enzimas metabólicas, sugiriendo una falla en la regulación de las rutas metabólicas (Guerrero-Zárate et al., 2019). Esto probablemente se deba a un daño hepático causado por una acumulación excesiva de lípidos en los hepatocitos como fue evidenciado a través de análisis histológicos (datos no publicados). Lo mismo puede haber causado que los peces que recibieron la dieta 3 tuvieran los niveles de glucosa más altos durante la prueba de tolerancia a la glucosa.

También, es sabido que los experimentos de programación nutricional, la dosis y el periodo durante el cual se aplica el estímulo son relevantes, además de ser específicos para cada especie (Kamalam et al., 2017). En la presente investigación se puede observar que el suministrar una dieta con niveles elevados de carbohidratos (28\%) por un periodo prolongado de tiempo (73 días) puede causar un afecto permanente, semejante al de resistencia a la insulina en el pejelagarto. Demostrado por la hiperglicemia en los peces que recibieron la D3, aun después de que los organismos experimentales ya estaban consumiendo una dieta comercial y había sido sometidos a $24 \mathrm{~h}$ de ayuno previo a la prueba de GTT. Por lo que proponemos que existe un nivel de CHO y un tiempo de exposición umbral que no deben ser traspasados durante el periodo de programación nutricional a fin de no causar un daño permanente a los peces.

Por otro lado, la concentración de triglicéridos en plasma mostró diferencias altamente significativas $(\mathrm{P}<0.0001)$ debidas al tipo de dieta recibida, sin embargo, no se observaron diferencias estadísticas debidas al tiempo después de la aplicación de la dextrosa $(\mathrm{P}=0.3510)$. La concentración de triglicéridos en plasma fue mayor en los 
peces alimentados con la D1, seguidos de los alimentados con la D2, observándose la menor concentración en los peces alimentados con la D3 (Figura 3).

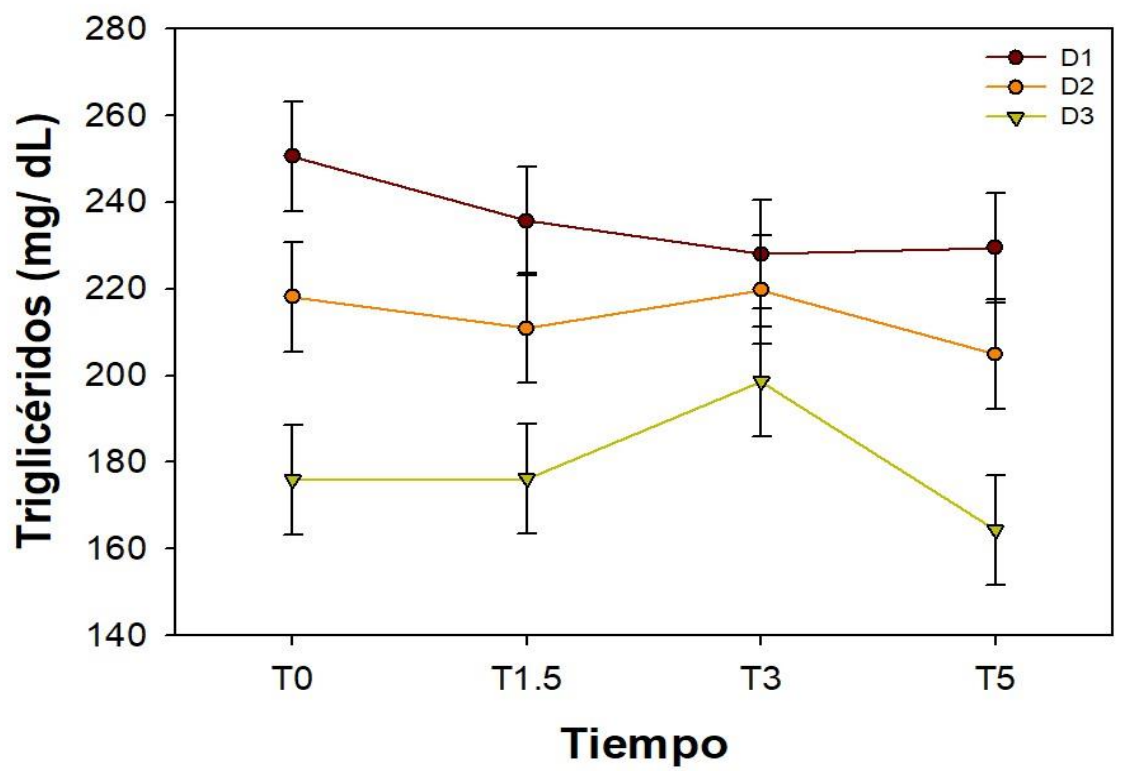

Figura 3. Niveles de triglicéridos en plasma después de la administración de $1.2 \mathrm{~g}$ de dextrosa/kg de peso. Media $\pm E E, n=9$

La concentración del colesterol en plasma no se vio significativamente afectada ni por la dieta suministrada $(\mathrm{P}=0.2375)$ ni por el tiempo transcurrido después de la aplicación del tratamiento $(\mathrm{P}=0.1260)$ (Figura 4$)$.

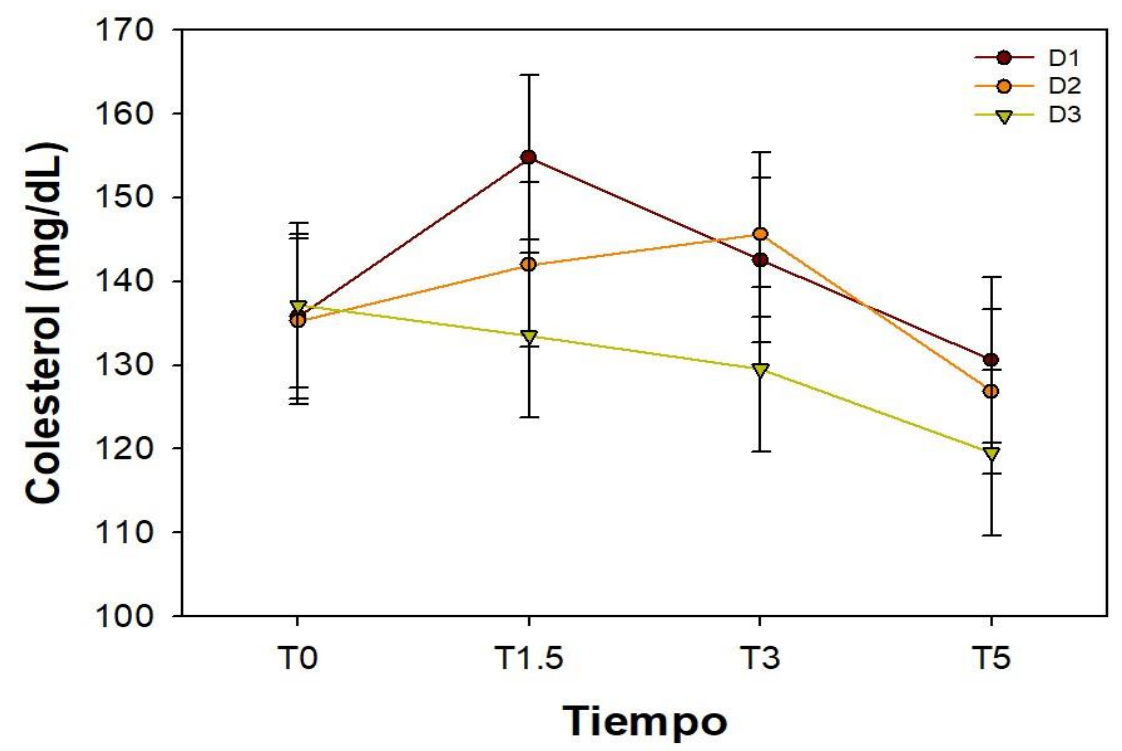

Figura 4. Niveles de colesterol en plasma después de la administración de $1.2 \mathrm{~g}$ de dextrosa/kg de peso. Media $\pm E E, n=9$ 
Lo que sugiere que la diferencia en la concentración en plasma obtenida después de la prueba de GTT se debe a la dieta que recibieron durante la etapa de crianza, mientras que la prueba de tolerancia a la glucosa no tuvo ningún efecto sobre estos parámetros. Una investigación anterior en pejelagarto pone en evidencia estas diferencias mientras los peces eran alimentados con diferentes proporciones de $\mathrm{CHO} /$ lípidos (L), donde la concentración de triglicéridos se incrementa mientras disminuye de proporción CHO/L, es decir, cundo hay más lípidos en la dieta. Los resultados de la presente investigación demuestran que el efecto de dietas ricas en lípidos administradas en etapas de larvicultivo permanece hasta la etapa juvenil, a pesar de que los peces de los tres grupos reciban la misma dieta posteriormente (dieta comercial). En el caso del colesterol, sus concentraciones no se vieron afectadas por la dieta suministrada durante la etapa de crianza ni por la prueba de tolerancia a la glucosa.

\section{CONCLUSIÓN}

En conclusión, la concentración de carbohidratos y lípidos suministrada durante la etapa temprana de crianza puede modificar de forma permanente las concentraciones de glucosa y triglicéridos en plasma, así como la tolerancia a la glucosa de los pejelagartos juveniles. Lo que sugiere que puede existir un afecto de programación metabólica.

\section{AGRADECIMIENTOS}

Los autores agradecen al Programa para el Desarrollo Profesional Docente (PRODEP) por el apoyo brindado para el desarrollo de esta investigación a través del financiamiento del proyecto "programación nutricional en el pejelagarto (Atractosteus tropicus) y su impacto en el metabolismo intermediario" UJAT-EXB-243

\section{REFERENCIAS BIBLIOGRAFICAS}

Enes, P., Panserat, S., Kaushik, S., \& Oliva-Teles, A. (2006). Effect of normal and waxy maize starch on growth, food utilization and hepatic glucose metabolism in European sea bass (Dicentrarchus labrax) juveniles. Comparative Biochemistry and Physiology - A Molecular and Integrative Physiology, 143(1), 89-96. https://doi.org/10.1016/j.cbpa.2005.10.027

Enes, P., Panserat, S., Kaushik, S., \& Oliva-Teles, A. (2008). Growth performance and metabolic utilization of diets with native and waxy maize starch by gilthead sea bream (Sparus aurata) juveniles. Aquaculture, 274(1), 101-108. https://doi.org/10.1016/j.aquaculture.2007.11.009 
Fang, L., Liang, X. F., Zhou, Y., Guo, X. Z., He, Y., Yi, T. L., Liu, L.W., Yuan, X.C. \& Tao, Y. X. (2014). Programming effects of high-carbohydrate feeding of larvae on adult glucose metabolism in zebrafish, Danio rerio. British Journal of Nutrition, 111(5), 808-818. https://doi.org/10.1017/S0007114513003243

Folch, J., Lees, M., \& Sloan Stanley, G. (1957). A simple method for the isolation and purification of total lipides from animal tissues. The Journal of Biological Chemistry, 226, 497-509. https://doi.org/10.1016/j.ultrasmedbio.2011.03.005

Frías-Quintana, C., Álvarez-González, C., \& Márquez-Couturier, G. (2010). Diseño de microdietas para el cultivo de pejelagarto Atractosteus tropicus, Gill 1863. Universidad y Ciencia, 26(2), 265-282.

Frías-Quintana, C., Domínguez-Lorenzo, J., Álvarez-González, C., Tovar-Ramírez, D., \& Martínez-García, R. (2016). Using cornstarch in microparticulate diets for larvicultured tropical gar (Atractosteus tropicus). Fish Physiology and Biochemistry, 42(2), 517-528. https://doi.org/10.1007/s10695-015-0156-4

Gong, G., Xue, M., Wang, J., Wu, X. F., Zheng, Y. H., Han, F., Liang, X. F \& Su, X. O. (2015). The regulation of gluconeogenesis in the Siberian sturgeon (Acipenser baerii) affected later in life by a short-term high-glucose programming during early life. Aquaculture, 436, 127-136. https://doi.org/10.1016/j.aquaculture.2014.10.044

Guerrero-Zárate, R., Álvarez-González, C., Jesus-Contreras, R., Peña-Marín, E., Martínez-García, R., Galaviz, M., López L.M. \& Llera-Herrera, R. (2019). Evaluation of carbohydrate / lipid ratios on growth and metabolic response in tropical gar ( Atractosteus tropicus ) juvenile. Aquaculture Research, 50, 18121823. https://doi.org/10.1111/are.14060

Guerrero-Zárate, R., Alvarez-González, C., Olvera-Novoa, M., Perales-García, N., Frías-Quintana, C., Martínez-García, R., \& Contreras-Sánchez, W. (2014). Partial characterization of digestive proteases in tropical gar Atractosteus tropicus juveniles. Fish Physiology and Biochemistry, 40(4), 1021-1029. https://doi.org/10.1007/s10695-013-9902-7

Hemre, G.-I., Mommsen, T. P., \& Krogodahl, A. (2002). Carbohydrates in fish nutrition: effects on growth, glucose metabolism and hepatic enzymes. Aquaculture Nutrition, 8(3), 175-194. https://doi.org/10.1046/j.1365- 
2095.2002.00200.x

Hou, Z., \& Fuiman, L. A. (2019). Nutritional programming in fishes : insights from mammalian studies. Reviews in Fish Biology and Fisheries, 30(5), 67-92. https://doi.org/10.1007/s11160-019-09590-y

Kamalam, B., Medale, F., \& Panserat, S. (2017). Utilisation of dietary carbohydrates in farmed fishes: New insights on influencing factors, biological limitations and future $\quad$ strategies. $\quad$ Aquaculture, $\quad 467, \quad 3-27$. https://doi.org/10.1016/j.aquaculture.2016.02.007

Kamalam, B., \& Panserat, S. (2016). Carbohydrates in fish nutrition. International AquaFeed, April, 20-23. https://doi.org/10.13140/RG.2.1.4570.6645

Liang, X., Wang, J., Gong, G., Xue, M., Dong, Y., Wu, X., Wang, X., Chen, C. Liang, X. \& Qin, Y. (2017). Gluconeogenesis during starvation and refeeding phase is affected by previous dietary carbohydrates levels and a glucose stimuli during early life in Siberian sturgeon (Acipenser baerii). Animal Nutrition, 3(3), 284294. https://doi.org/10.1016/j.aninu.2017.06.001

Márquez-Couturier, G., Vázquez-Navarrete, C., Contreras-Sánchez, W., \& AlvarezGonzález, C. (2015). Acuicultura tropical sustentable: Una estrategia para la producción y conservación del pejelagarto (Atractosteus tropicus) en Tabasco, México $\left(2^{\mathrm{a}}\right)$. Villahermosa, Tabasco: Universidad Juárez Autónoma de Tabasco. http://publicaciones.ujat.mx/cientificas/Documentos/Biología/36/Acuicultura tropical sustentable 11.pdf

Panserat, S., Marandel, L., \& Seiliez, I. (2019). New insights on intermediary metabolism for a better understanding of nutrition in teleosts. Annual Review of Animal Bioscience, 7, 195-220. https://doi.org/10.1146/annurev-animal-020518115250

Panserat, S., Médale, F., Blin, C., Brèque, J., Vachot, C., Plagnes-Juan, E., Gomes, E., Krishnamoorthy, R. \& Kaushik, S. (2000). Hepatic glucokinase is induced by dietary carbohydrates in rainbow trout, gilthead seabream, and common carp. American Journal of Physiology-Regulatory, Integrative and Comparative Physiology, 278, 1164-1170.

Panserat, S., Médale, F., Brèque, J., Plagnes-Juan, E., \& Kaushik, S. (2000). Lack of significant long-term effect of dietary carbohydrates on hepatic glucose-6- 
phosphatase expression in rainbow trout (Oncorhynchus mykiss). The Journal of Nutritional Biochemistry, 11(1), 22-29. https://doi.org/10.1016/S09552863(99)00067-4

Polakof, S., Mommsen, T. P., \& Soengas, J. L. (2011). Glucosensing and glucose homeostasis: From fish to mammals. Comparative Biochemistry and Physiology - B Biochemistry and Molecular Biology, 160(4), 123-149. https://doi.org/10.1016/j.cbpb.2011.07.006

Polakof, S., Panserat, S., Soengas, J. L., \& Moon, T. W. (2012). Glucose metabolism in fish: A review. Journal of Comparative Physiology B: Biochemical, Systemic, and Environmental Physiology, 182(8), 1015-1045. https://doi.org/10.1007/s00360-012-0658-7

Polakof, S., Skiba-Cassy, S., \& Panserat, S. (2009). Glucose homeostasis is impaired by a paradoxical interaction between metformin and insulin in carnivorous rainbow trout. American Journal of Physiology - Regulatory Integrative \& Comparative Physiology, 297, R1769-R1776. https://doi.org/10.1152/ajpregu.00369.2009.

Thivend, P., Mercier, C., \& Guilbot, A. (1972). Determination of starch with glucoamylase. In R. Whistler \& J. Bemiller (Eds.), General Carbohydrate Method (pp. 100-105). New York, USA: Academic Press. https://doi.org/10.1016/b978-0-12-746206-6.50021-7

William Horwitz. (2000). Official methods of analysis of AOAC International. Association of Official Analytical Chemists (17th ed.). Gaithersburg, MD, USA.

Wilson, R. P. (1994). Utilization of dietary carbohydrate by fish. Aquaculture, 124(14), 67-80. https://doi.org/10.1016/0044-8486(94)90363-8 